\title{
Detection of the phosphatase activity of carbonic anhydrase III on a nitrocellulose membrane following 2D gel electrophoresis
}

\author{
HE HUANG ${ }^{1,2}$, HUI-MIN REN ${ }^{1}$, XI-LIANG SHANG ${ }^{3}$ and XUE-YUAN LIU ${ }^{2}$ \\ ${ }^{1}$ Institute of Neurology, Fudan University, Shanghai 200040; ${ }^{2}$ Department of Neurology, \\ Shanghai Tenth People's Hospital of Tongji University, Shanghai 200092; ${ }^{3}$ Department of Sports Medicine, \\ Fudan University, Shanghai 200040, P.R. China
}

Received November 11, 2013; Accepted May 14, 2014

DOI: $10.3892 / \mathrm{mmr} .2014 .2439$

\begin{abstract}
Carbonic anhydrase isozyme III (CAIII) is unique among the carbonic anhydrases because it exhibits phosphatase activity. CAIII is relatively specific to skeletal muscles, and may therefore be a useful diagnostic marker for muscular diseases. In the muscles of patients with myasthenia gravis (MG), CAIII is deficient and previous studies have demonstrated that changes in the phosphatase activity of CAIII is a fundamental mechanism underlying the weakness and fatigability of MG. However, there have been no effective analytical methods for investigating its phosphatase activity until now. In the present study, a new method combining two-dimensional electrophoresis (2-DE) and phosphatase staining in situ on a nitrocellulose membrane was reported to detect the phosphatase of CAIII in skeletal muscle extracts. Furthermore, a recombinant CAIII was constructed and its phosphatase activity staining was demonstrated to be positive. This method allows for the effective detection of the phosphatase activity of CAIII following 2-DE and is a promising technique for functional proteomics.
\end{abstract}

\section{Introduction}

Recently, carbonic anhydrase III (CAIII), a member of the carbonic anhydrase family, was reported to be insufficient in the skeletal muscles of patients with myasthenia gravis (MG) (1). There are at least 16 proteins containing the carbonic anhydrase structure, of which 12 proteins function as hydratase (2). By contrast with other isozymes, CAIII is distinguished by several characteristics. It is expressed

Correspondence to: Mr. He Huang or Dr Hui-Min Ren, Institute of Neurology, Fudan University, 12 Middle Wulumuqi Road, Shanghai 200040, P.R. China

E-mail: morton162@hotmail.com

E-mail: renhm188@163.com

Key words: carbonic anhydrase III, phosphatase, activity staining, 2D gel electrophoresis, recombination selectively in skeletal muscle (comprising $8 \%$ of the soluble protein), the liver (5\%) and in adipocytes (25\%) (3-5). Except for a lower hydratase activity ( $1 \%$ of the isozyme II) and an esterase activity, it was reported that CAIII has phosphatase-related activities (6).

As revealed by in vitro studies in skeletal muscle, CAIII is involved in cellular oxidative stress, apoptosis and signal transduction (7-9). As attributed to the presence of reactive cysteine residues in its structure, CAIII is reversibly $\mathrm{S}$-glutathiolated to modulate the process of oxidation-deoxidation $(8,10)$ and simultaneously, its phosphatase activity may be modified by S-glutathiolation. Therefore, it is hypothesized that CAIII may act as a regulator of the phosphorylation processes in cell signaling, although the endogenous substrate of CAIII phosphatase has yet to be elucidated.

Previous studies have demonstrated that the activity of CAIII was dependent on glutathiolation, which may be affected by the processes of separation and purification of CAIII $(6,11)$. Theoretically, methods that directly detect CAIII phosphatase activity against cell extracts may allow simple and efficient analysis of skeletal muscle in disease pathogenesis. The present study describes a novel method, combining two-dimensional electrophoresis (2-DE) and phosphatase staining in situ on a nictrocellulose membrane, to detect the phosphatase activity of CAIII in skeletal muscle extracts. Furthermore, a recombinant CAIII was produced for further validation.

\section{Materials and methods}

Ethical considerations. All procedures performed on the rat were in accordance with the EU (86./609/EEC) for the care and use of laboratory animals.

Preparation of rat skeletal muscle homogenate. Male adult Sprague-Dawley rats, weighing 150-180 g, were obtained from The Experimental Animal Center of Shanghai Medical College, Fudan University (Shanghai, China). All experiments were conducted according to the guidelines of the Animal Care Committee of Fudan University. All reagents were purchased from Sinopharm Chemical Reagent Co., Ltd. (Shanghai, China) unless otherwise noted. After anaesthetizing the rats with an 
intraperitoneal injection of pentobarbitol $(50 \mathrm{mg} / \mathrm{kg})$, the soleus muscles were dissected from the hind legs and homogenized in $10 \mathrm{mM}$ sodium phosphate buffer ( $\mathrm{pH}$ 7.4), containing a mixture of protease inhibitors. The homogenate was centrifuged at $10,000 \times \mathrm{g}$ at $4^{\circ} \mathrm{C}$ for $20 \mathrm{~min}$, and then the supernatant was collected. The protein concentration was determined by Bradford's method with bovine serum albumin as standard.

2-DE and immunoblotting. A symmetric miniature 2-DE was performed (12). In brief, $6 \%$ isoelectric focusing (IEF) gels with a diameter of $1.2 \mathrm{~mm}$ and a length of $60 \mathrm{~mm}$, containing $1 \%$ (v/v) pH 3.5-10 ampholine, 1\% (v/v) pH 5-8 ampholine (Sigma, St. Louis, MO, USA) and $2 \%(\mathrm{v} / \mathrm{v})$ glycerol, were prepared. To each gel, $30 \mu \mathrm{g}$ of protein was added and two gels were electrofocused simultaneously at $200 \mathrm{~V}$ for $20 \mathrm{~min}$ and then $400 \mathrm{~V}$ for $5 \mathrm{~h}$. The anolyte and catholyte contained $20 \mathrm{mM}$ $\mathrm{NaOH}$ and $10 \mathrm{mM} \mathrm{H}_{3} \mathrm{PO}_{4}$, respectively. The gels were equilibrated with buffer for the second-dimensional sodium dodecyl sulfate-polyacrylamide gel electrophoresis (SDS-PAGE) at room temperature and submitted to electrophoresis on a $10 \%$ SDS-PAGE gel at $30 \mathrm{~V}$ for $20 \mathrm{~min}$, then at $100 \mathrm{~V}$ until the bromophenol blue dye reached the end.

SDS-PAGE-separated proteins were transferred onto a nitrocellulose membrane. The membrane was divided into two parts, one of which was selected randomly for protein staining by ponceau red $\mathrm{S}$ and the other for CAIII immunodetection by a polyclonal antibody from rabbit against rat skeletal CAIII, at a dilution of 1:1,600.

Phosphatase activity staining in situ. Following 2-DE, the proteins were transferred to a nitrocellulose membrane in an ice bath. Once divided into halves, one half of the membrane selected for enzyme staining was incubated in ABS buffer containing $20 \mathrm{mM}$ sodium acetate, $0.8 \%$ (w/v) sodium chloride and $0.02 \%(\mathrm{w} / \mathrm{v})$ potassium chloride $(\mathrm{pH} 5.5)$ at $25^{\circ} \mathrm{C}$ for $20 \mathrm{~min}$, to permit removal of SDS and refolding of the proteins. Then 5\% (w/v) polyvinylpyrrolidone (MW, 40,000 kDa; Sigma) in ABS buffer was applied to block the non-specific binding sites, at $4^{\circ} \mathrm{C}$ overnight. Following washing with the ABS buffer for $5 \mathrm{~min}$, the membrane was dipped into a $20 \mathrm{ml}$ staining system ( $\mathrm{pH} 5.5$ ) with final concentrations of $50 \mathrm{mM}$ sodium acetate, $20 \mathrm{mM}$ magnesium chloride, $5 \mathrm{mM}$ nitrophenyl phosphate (Fluka, Buchs, Switzerland), $2 \mathrm{mM}$ fast garnet GBC (Sigma) (13). The staining system was stopped at $37^{\circ} \mathrm{C}$ for $45-60 \mathrm{~min}$.

Expression of recombinant CAIII and its phosphatase activity. The CAIII gene (GenBank accession no. M22413) was designed using BamHI and HindIII sites and amplified, using the primers: primer 1 (5'-AGAGGATCCGAATTCGCTAAGGAGTGG GGTTAC-3') and primer 2, (5'-AGAAAGCTTCTTGAAGG AGGCCCTCACCAC-3'). The plasmid pET28a (Novagen, Darmstadt, Germany) and amplified DNA were digested with BamHI and HindIII, then ligated with T4 DNA ligase (Fermentas, Vilnius, Lithuania) and transformed into E. coli DH5a (Novagen, Darmstadt, Germany). The positive recombinant plasmids (pET28a-CAIII) were confirmed by restriction enzyme digestion and DNA sequencing (Invitrogen Biotechnology, Co., Ltd., Shanghai, China). The recombinant plasmids were then transformed into E. coli BL21 (DE3;
Novagen) for protein expression. The transformants were grown in Luria-Bertani medium (1.0\% tryptone, $1.0 \% \mathrm{NaCl}$, $0.5 \%$ yeast extract, $\mathrm{pH} 7.0$ ) containing $50 \mathrm{mg} / 1 \mathrm{kanamycin}$ at $37^{\circ} \mathrm{C}$. When the OD600 reached 0.9, IPTG was added to the final concentration of $1.0 \mathrm{mM}$. Following incubation at $37^{\circ} \mathrm{C}$ for $8 \mathrm{~h}$, the bacteria were collected and resuspended in buffer A (50 mM NaH $\mathrm{PO}_{4}, 10 \mathrm{mM}$ imidazole, $500 \mathrm{mM} \mathrm{NaCl}$, $10 \%$ glycerol, $1 \mathrm{mM}$ phenylmethanesulfonyl fluoride, $\mathrm{pH}$ 8.0) and disrupted by sonication on ice. The supernatant was obtained by centrifugation at $5,000 \mathrm{x}$ g for $20 \mathrm{~min}$ at $4^{\circ} \mathrm{C}$ and then loaded onto a Ni-NTA affinity chromatography column (Novagen). The column was washed with buffer B $(10 \mathrm{mM}$ Tris- $\mathrm{HCl}, 100 \mathrm{mM} \mathrm{NaH}_{2} \mathrm{PO}_{4}, 8 \mathrm{M}$ urea, $\mathrm{pH}$ 8.0) and buffer $\mathrm{C}$ (10 mM Tris- $\mathrm{HCl}, 100 \mathrm{mM} \mathrm{NaH} \mathrm{PO}_{4}, 8 \mathrm{M}$ urea, $\mathrm{pH}$ 6.9) to remove the unbound proteins and eluted with buffer $\mathrm{D}(10 \mathrm{mM}$ Tris- $\mathrm{HCl}, 100 \mathrm{mM} \mathrm{NaH} \mathrm{PO}_{4}, 8 \mathrm{M}$ urea, $\mathrm{pH}$ 4.5). The eluates were collected and analyzed by means of SDS-PAGE. The proteins were renaturated by urea gradient dialysis, then dialyzed against $0.01 \mathrm{M}$ phosphate-buffered saline ( $\mathrm{pH} 7.4)$ and subsequently analyzed by $10 \%$ SDS-PAGE and western blotting. The phosphatase activity was examined as mentioned above.

\section{Results}

Separation and identification of CAIII from skeletal muscle. Since the $\mathrm{pH}$ of CAIII has been reported as 6.8, 7.0 and $7.2(1,9)$, pI 5-8 ampholine (1\%, v/v) and pH 3.5-10 ampholine $(1 \%, \mathrm{v} / \mathrm{v})$ was used in isoelectric focusing for the separation of CAIII. In this procedure, two IEF strips were placed symmetrically on the secondary SDS-PAGE. A symmetric proteome map was developed and then transferred onto a nitrocellulose membrane. One half of the membrane was stained by ponceau red S (2-DE map; Fig. 1A) and the other for immunodetection of CAIII (Fig. 1B). According to the mirror image on the immunoblotting membrane (Fig. 1B), CAIII was identified and located on the 2-DE map (Fig. 1A).

Phosphatase activity staining of CAIII following $2 D \mathrm{gel}$ electrophoresis. For enzyme activity staining, the membrane containing symmetric proteome maps was divided into halves, one for ponceau red S staining (2-DE map; Fig. 2A) and the other for phosphatase staining. Using nitrophenyl phosphate as a reagent, a phosphatase staining map was obtained (Fig. 2B). Referring to the CAIII staining located on the 2-DE map by ponceau red S (Fig. 2A), the mirror CAIII spot, exhibiting active staining on the enzyme staining map was identified (Fig. 2B).

For further analysis, the correlation between the time and density of phosphatase staining was described. A plot of time vs. the density of phosphatase staining [optical density (OD) value] calculated by the software Image-Pro Plus 6.0 is presented in Table I and Fig. 3. A negative staining without substrate was performed simultaneously. The density of CAIII staining was dependent on the reaction time and the linear correlation maintained within 60 min (Fig. 3C).

Furthermore, the inhibition of phosphatase staining was examined. The inhibitors, potassium phosphate (1.94 mM) and sodium fluoride $(1.94 \mathrm{mM})$, were added separately into the CAIII phosphatase staining system. Fig. 4 demonstrated 


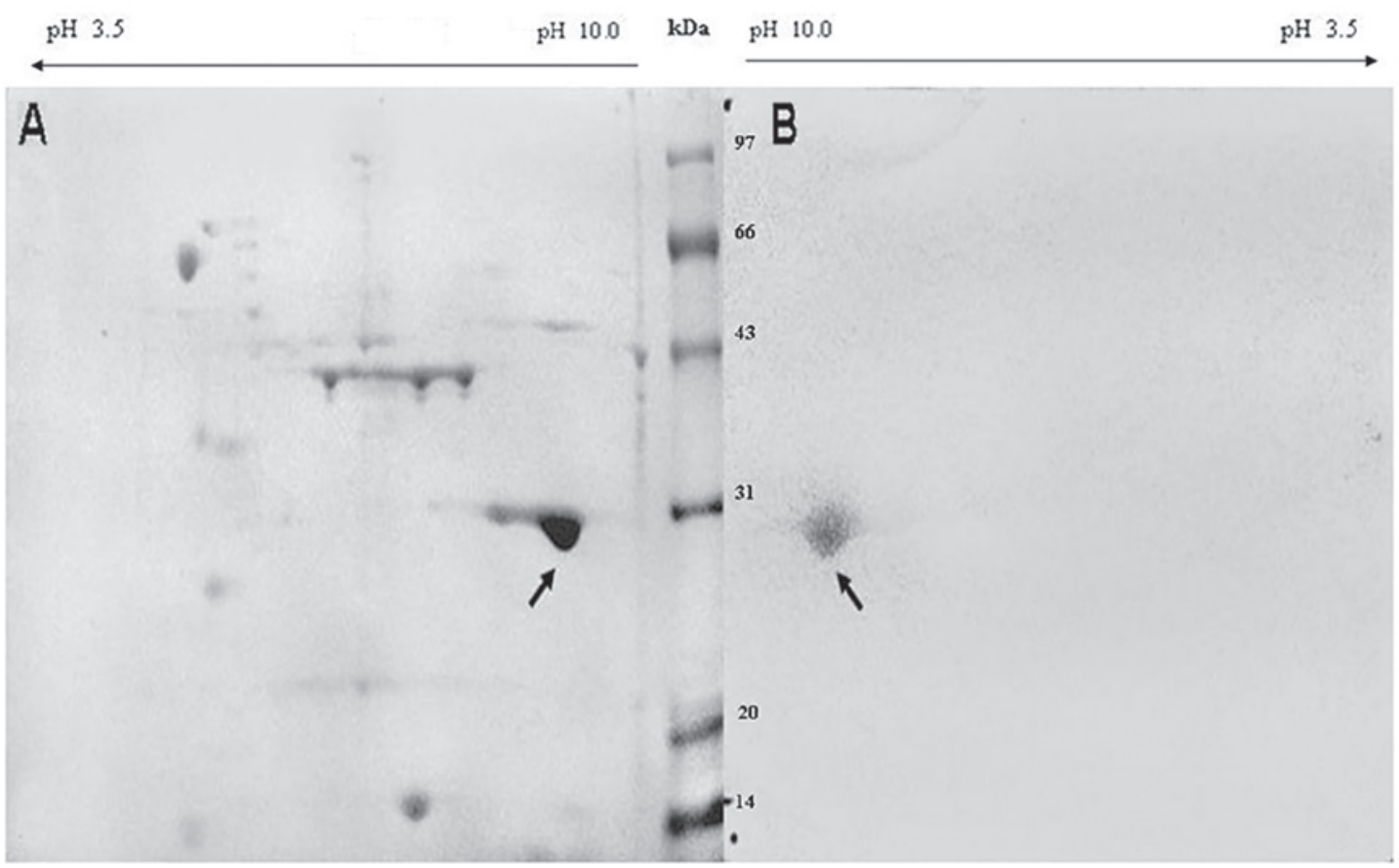

Figure 1. Immunoblotting of CAIII following 2-DE. (A) The skeletal muscle proteins were electrophoretically transferred onto a nitrocellulose membrane and stained by ponceau red S following 2-DE. (B) Immunodetection of CAIII by a specific antibody (dilution, 1:1,600). CAIII, carbonic anhydrase isozyme III; 2-DE, two-dimensional electrophoresis.

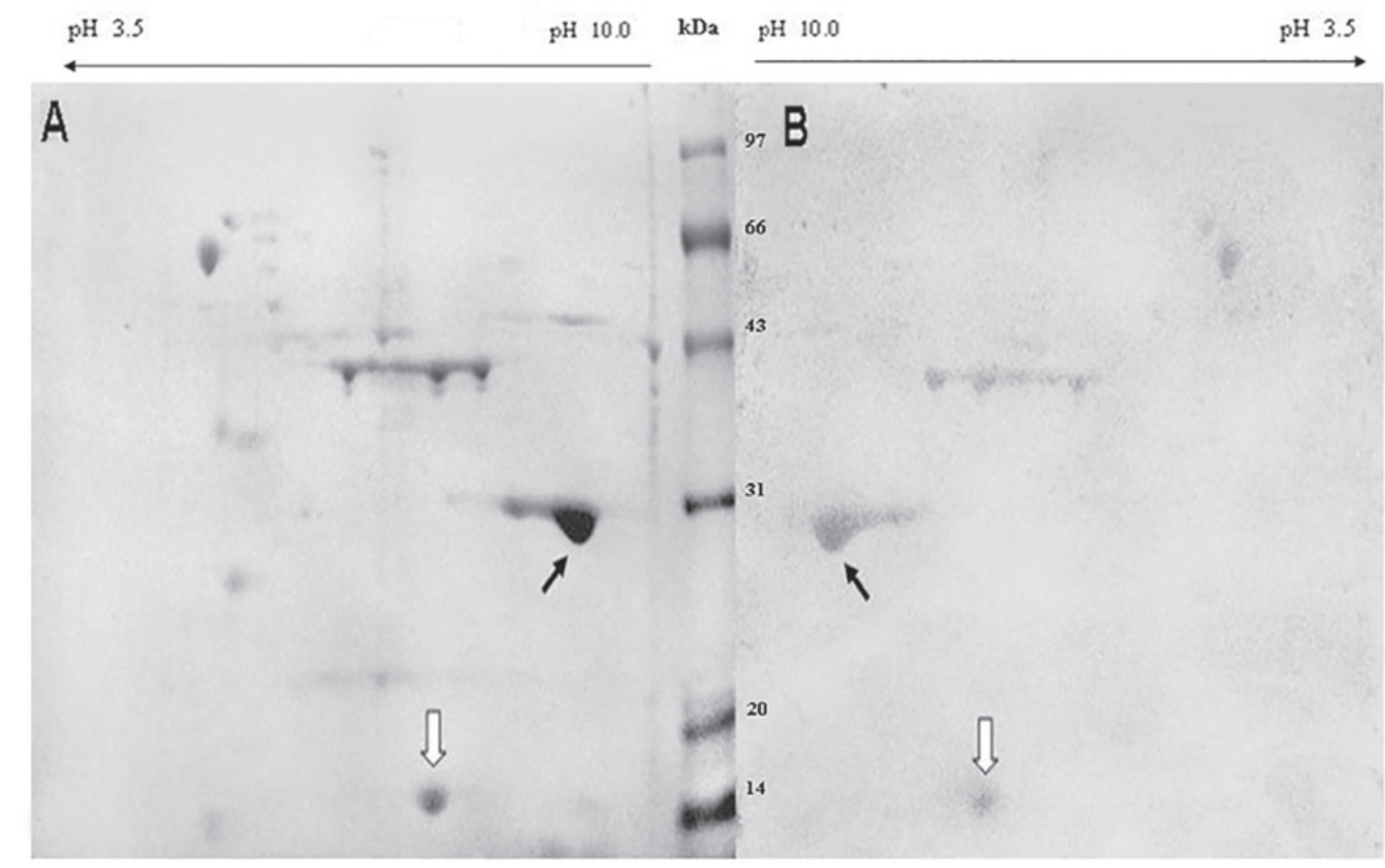

Figure 2. Phosphatase staining on nitrocellulose membrane following 2-DE. (A) Ponceau red S staining following transferring. (B) Phosphatase staining using the substrate nitrophenyl phosphate. CAIII and its phosphatase staining spot are indicated by the opaque arrows. CAIII, carbonic anhydrase isozyme III; 2-DE, two-dimensional electrophoresis.

that the phosphatase staining was suppressed by both inhibitors, particularly for potassium phosphate.

Expression of recombinant CAIII and its phosphatase activity. The plasmid pET28a and the amplified DNA digested by BamHI and HindIII were ligated to construct recombinant plasmids pET28a-CAIII. The constructed plasmids from the transformants of E. coli DH5a were confirmed by restriction enzyme digestion (Fig. 5) and DNA sequencing (data not presented). The confirmed recombinant plasmids 
A
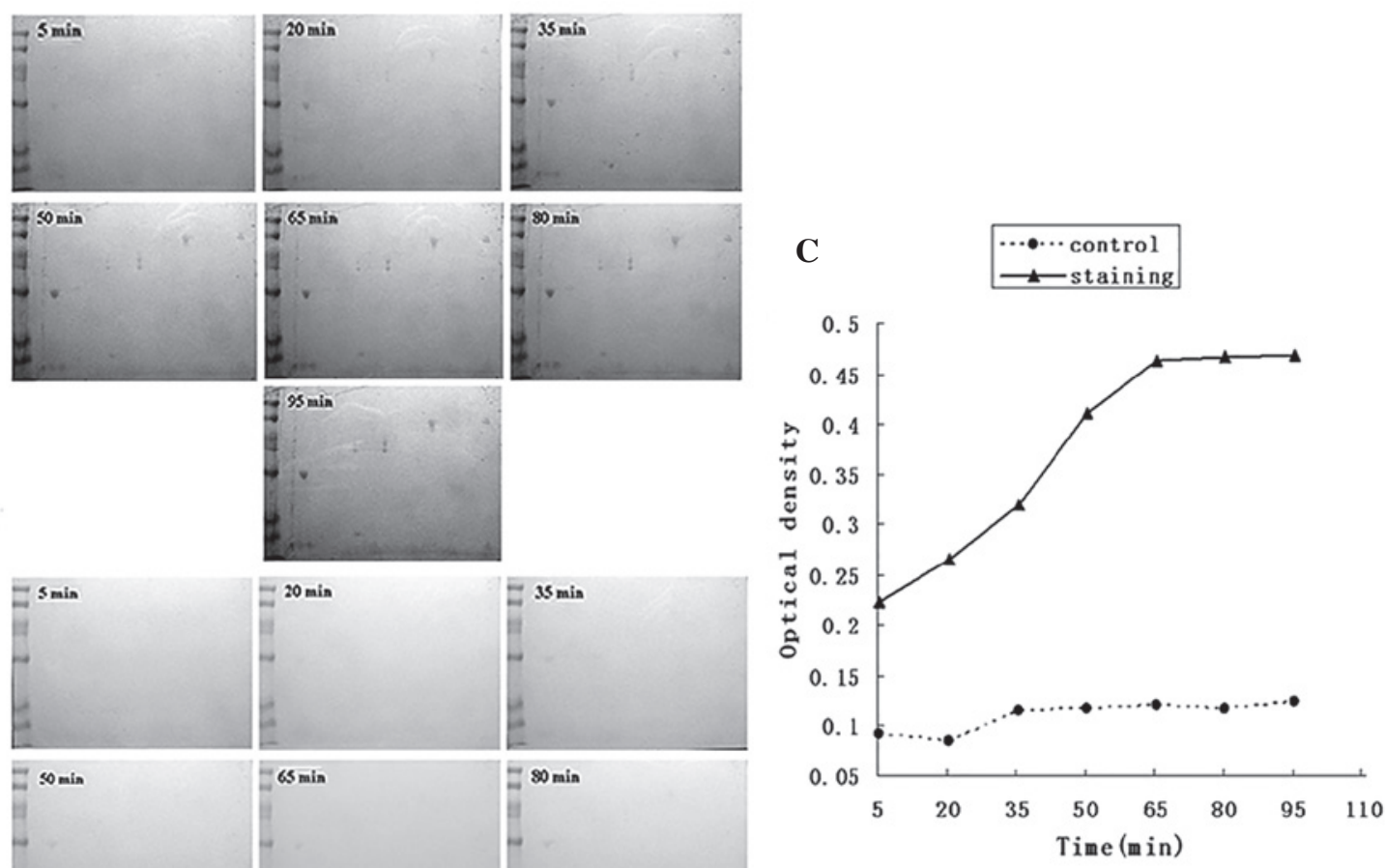

B
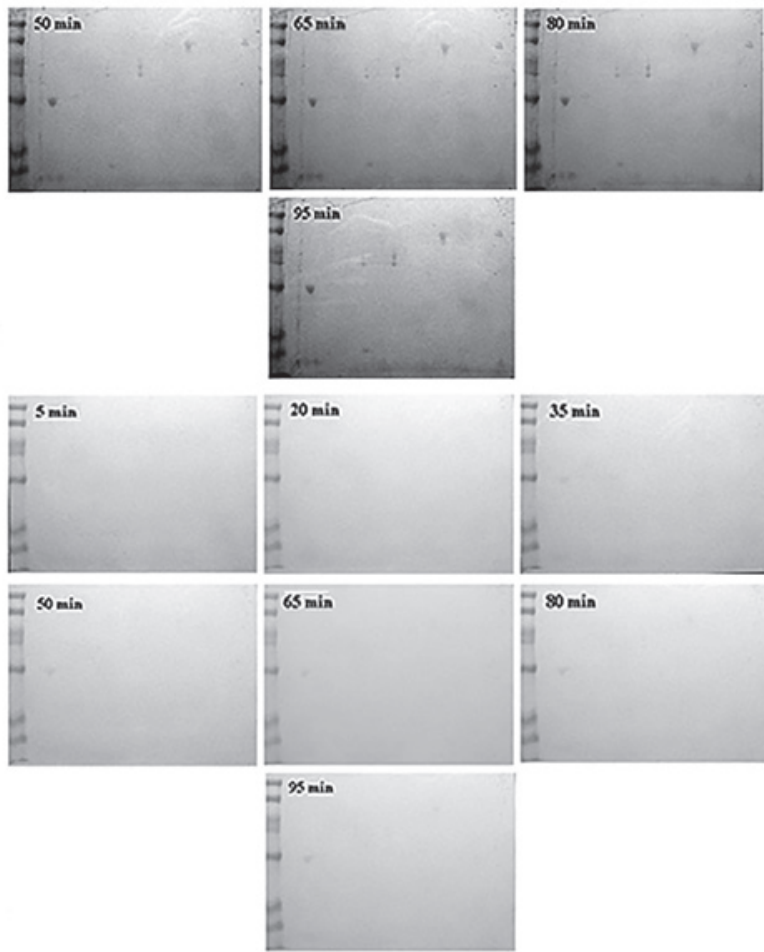

Figure 3. Correlation between the reaction time and product formation. The density of staining was quantified using the software Image-Pro Plus 6.0 at 0,15 , 30, 45, 60, 75 and $90 \mathrm{~min}$. The staining reactions, (A) with or (B) without the substrate nitrophenyl phosphate were performed, respectively. (C) Plot of time vs. the staining density, the solid line represents with the substrate and the dashed line without the substrate.

Table I. Correlation between reaction time and phosphatase staining of CAIII.

\begin{tabular}{lcc}
\hline Time (min) & $\begin{array}{c}\text { Control } \\
\text { (OD value) }\end{array}$ & $\begin{array}{c}\text { CAIII } \\
\text { (OD value) }\end{array}$ \\
\hline 5 & 0.092 & 0.238 \\
20 & 0.085 & 0.265 \\
35 & 0.114 & 0.319 \\
50 & 0.116 & 0.411 \\
65 & 0.120 & 0.463 \\
80 & 0.117 & 0.466 \\
95 & 0.123 & 0.469
\end{tabular}

The density of staining (OD value) was quantified using the software Image-Pro Plus 6.0 at $0,15,30,45,60,75$ and $90 \mathrm{~min}$. The staining reactions without the substrate were performed as the controls. CAIII, carbonic anhydrase isozyme III; OD, optical density.

were transformed into E. coli BL21 (DE3) for protein expression. Following induction by IPTG, the fusion proteins were harvested and purified subsequently with a Ni-NTA affinity chromatography column, and then analyzed by SDS-PAGE and western blot analysis. Its activity was examined by phosphatase staining following SDS-PAGE, revealing active staining (Fig. 5).

\section{Discussion}

Currently, enzyme histochemistry, spectrophotometry and activity analysis following native-PAGE or SDS-PAGE are the most commonly applied methods for enzyme analyses under different conditions. Spectrophotometry is the classic method for quantitative determination of enzyme activity. However, this method firstly requires separation and purification of the specific enzyme from the complex, which is a time-consuming and complicated process. Furthermore, the obtained protein may be not be fully purified due to contamination with components of similar traits or changes of molecular structure, which reduces the validity of analysis and even leads to opposite conclusions. It has been reported that, following a modified procedure, the phosphatase activity of CAIII previously considered to be intrinsic was actually found to be extrinsic (11).

Theoretically, analytical methods to directly detect CAIII phosphatase activity against cellular extracts may facilitate a simple and efficient analysis of skeletal muscle. Activity analysis in situ following electrophoresis and the detection of enzyme activity following SDS-PAGE or native-PAGE is available and has been applied to expand functional proteomics by 

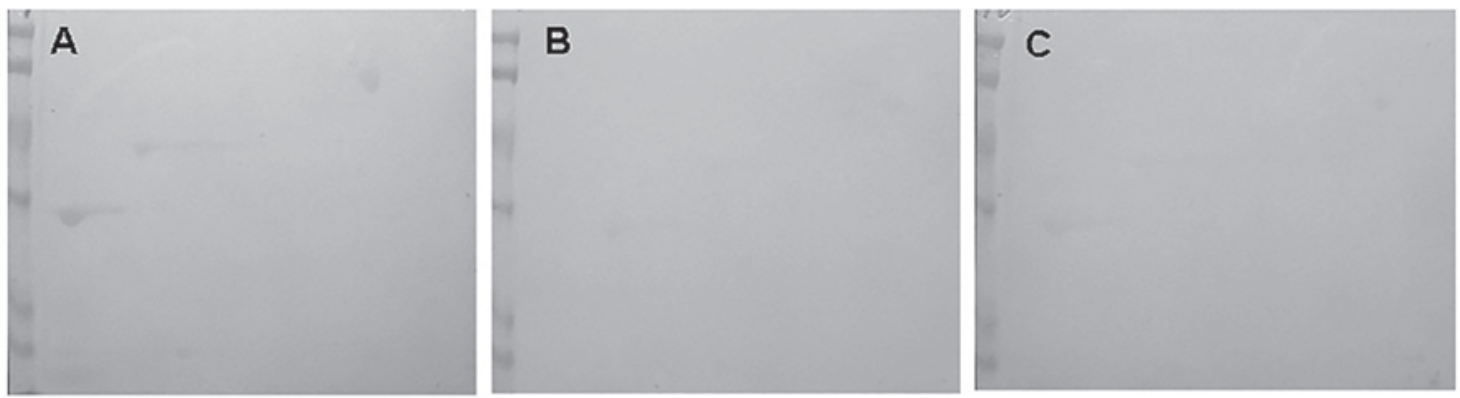

Figure 4. The inhibition effects of different agents on CAIII phosphatase activity. (A) Negative control or (B) $1.94 \mathrm{mM}$ of potassium phosphate or (C) $1.94 \mathrm{mM}$ of sodium fluoride was added separately into the CAIII phosphatase staining system. The phosphatase activity was examined as described in the Materials and methods section. CAIII, carbonic anhydrase isozyme III.
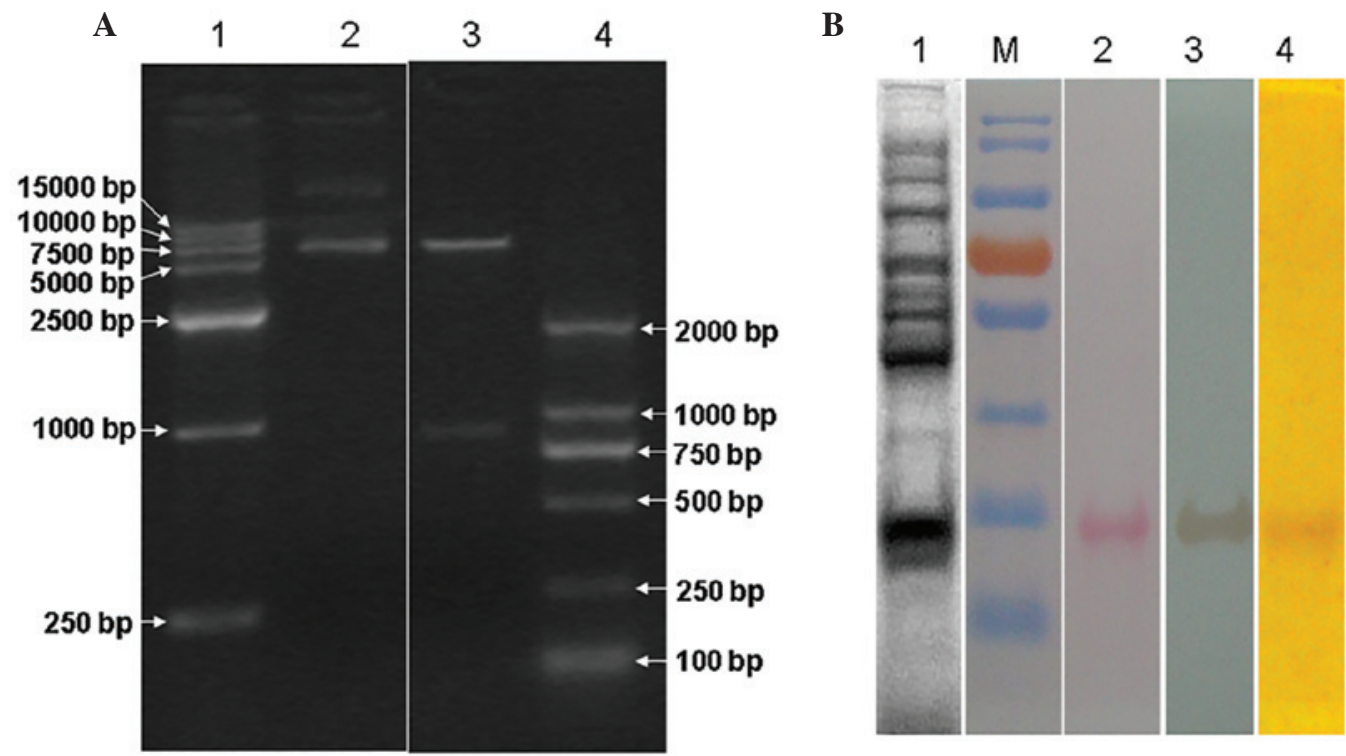

Figure 5. Expression of recombinant CAIII and its phosphatase staining. (A) Identification of recombinant plasmids pET28a-CAIII (1\% agarose gel electrophoresis); lane 1, DNA marker; lane 2, recombinant plasmid pET28a-CAIII; lane 3, digested recombinant plasmid pET28a-CAIII; lane 4, DNA marker. (B) Western blotting and phosphatase staining of recombinant CAIII. Lane M, prestained protein molecular weight marker; lane 1, the protein expression of E. coli BL21 (DE3), analyzed by SDS-PAGE; lane 2, eluates collected following Ni-NTA affinity chromatography column and analyzed by SDS-PAGE and ponceau red S staining; lane 3, immunoblotting of recombinant CAIII by an specific antibody against CAIII; lane 4, phosphatase staining of recombinant CAIII. CAIII, carbonic anhydrase isozyme III; SDS-PAGE, sodium dodecyl sulfate-polyacrylamide gel electrophoresis.

employing 2-DE. For example, it has been reported by Todaka and Kanekatsu (14) that $\beta$-amylase isozymes in dehydrated cucumber cotyledons was detected following 2-DE using this analytical method. The present study reported an novel analytical method for detecting and verifying the CAIII phosphatse activity in skeletal muscle adopting 2-DE.

In order to achieve separation of the CAIII, the most suitable ampholytes and PAGE concentration were determined according to the $\mathrm{pI}$ and molecular weight of CAIII. For IEF, a mixture of equal volumes of ampholine $\mathrm{pH} 3.5-10$ and ampholine $\mathrm{pH}$ 5-8 was constructed to guarantee separation of CAIII from the other proteins. Two IEF gels were loading symmetrically besides the loading slot on the top of SDS-PAGE. Following the simultaneous separation of the two protein samples by 2-DE, mirror 2-DE maps were obtained by protein staining. Then, immunodetection with an antibody against CAIII and LTQ mass spectrometry were used to verify the separation of CAIII. Furthermore, CAIII was located in 2-DE map according to the symmetric immunostaining (Fig. 1).

For phosphatase analysis, to facilitate the enzyme-substrate interaction, the proteins were transferred onto the nitrocellulose membrane following electrophoresis (15), which simultaneously facilitated reducing the inhibition of SDS on CAIII activity. PVP, a macromolecular compound that has been effectively used to detect glycoproteins (16), was selected as a blocking agent, which avoids the effects of the conventional blocking agent, including BSA, fetal calf serum and skimmed milk powder, which may be mixed with a variety of phosphatases, which subsequently affects the background staining. Similarly, the two symmetrical 2-DE maps following transferring were divided, one part for ponceau staining and the other for phosphatase activity staining. Based on the location of CAIII, as previously identified on the 2-DE map, it was possible to directly identify the active staining of CAIII on the phosphatase staining map. 
In order to verify the phosphatase staining from the non-specific staining, a negative control was set and the impact of the selected inhibitors, potassium phosphate and sodium fluoride (17), on CAIII phosphate phosphatase activity staining was examined. The results demonstrated that the phosphatase activity staining was significantly inhibited. In view of the enzyme catalysis, the time-phosphatase staining correlation was observed. The results revealed that the staining changed with the reaction time, demonstrating a strong linear association between the staining and reaction time within $65 \mathrm{~min}$, as is consistent with previous studies (6). In combination with image analysis software, this method may also allow a quantitative analysis of CAIII phosphate. Additionally, Rt-CAIII was constructed and phosphatase activity staining, was performed according to the methods described in the present study, and positive staining was demonstrated.

In conclusion, symmetrical 2-DE according to the physiochemical traits of CAIII, combined immunodetection with specific antibody against CAIII for separation and location, and phosphatase activity staining on a nitrocellulose membrane were performed to achieve the intuitive and highly accurate analysis of CAIII phosphatase in situ, and even for quantitative analysis. It is therefore hypothesized that the phosphatase activity analysis of CAIII following 2-DE is a reliable example of a promising technique for functional proteomics.

\section{Acknowledgements}

This study was supported by the Natural Science Foundation of China (no. 30170329).

\section{References}

1. Du AL, Ren HM, Lu CZ, Tu JL, Xu CF and Sun YA: Carbonic anhydrase III is insufficient in muscles of myasthenia gravis patients. Autoimmunity 42: 209-215, 2009.

2. Imtaiyaz Hassan M, Shajee B, Waheed A, Ahmad F and Sly WS: Structure, function and applications of carbonic anhydrase isozymes. Bioorg Med Chem 21: 1570-1582, 2013.
3. Pullan LM and Noltmann EA: Discovery, characterization, and study of some inhibition properties of two mammalian muscle carbonic anhydrases III. Ann NY Acad Sci 429: 152-164, 1984.

4. Spicer SS, Ge ZH, Tashian RE, Hazen-Martin DJ and Schulte BA: Comparative distribution of carbonic anhydrase isozymes III and II in rodent tissues. Am J Anat 187: 55-64, 1990.

5. Harju AK, Bootorabi F, Kuuslahti M, Supuran CT and Parkkila S: Carbonic anhydrase III: a neglected isozyme is stepping into the limelight. J Enzyme Inhib Med Chem 28: 231-239, 2013.

6. Koester MK, Pullan LM and Noltmann EA: The p-nitrophenyl phosphatase activity of muscle carbonic anhydrase. Arch Biochem Biophys 211: 632-642, 1981.

7. Roy P, Reavey E, Rayne M, Roy S, Abed El Baky M, Ishii Y and Bartholomew $\mathrm{C}$ : Enhanced sensitivity to hydrogen peroxide-induced apoptosis in Evil transformed Rat1 fibroblasts due to repression of carbonic anhydrase III. FEBS J 277: 441-452, 2010.

8. Räisänen SR, Lehenkari P, Tasanen M, Rahkila P, Härkönen PL and Väänänen HK: Carbonic anhydrase III protects cells from hydrogen peroxide-induced apoptosis. FASEB J 13: 513-522, 1999.

9. Dai HY, Hong CC, Liang SC, Yan MD, Lai GM, Cheng AL and Chuang SE: Carbonic anhydrase III promotes transformation and invasion capability in hepatoma cells through FAK signaling pathway. Mol Carcinog 47: 956-963, 2008.

10. Cabiscol E and Levine RL: The phosphatase activity of carbonic anhydrase III is reversibly regulated by glutathiolation. Proc Natl Acad Sci USA 93: 4170-4174, 1996.

11. Kim G, Selengut J and Levine RL: Carbonic anhydrase III: the phosphatase activity is extrinsic. Arch Biochem Biophys 377: 334-340, 2000.

12. Ren H, Xiao Y and Zhou Z: Discordance of increase in stress proteins in right and left myocardium of perfused isolated rat heart. Zhongguo Ying Yong Sheng Li Xue Za Zhi 13: 143-147, 1997 (In Chinese).

13. Kanellis AK, Solomos T and Mattoo AK: Visualization of acid phosphatase activity on nitrocellulose filters following electroblotting of polyacrylamide gels. Anal Biochem 179: 194-197, 1989.

14. Todaka D and Kanekatsu M: Analytical method for detection of beta-amylase isozymes in dehydrated cucumber cotyledons by using two-dimensional polyacrylamide gel electrophoresis. Anal Biochem 365: 277-279, 2007.

15. Bischoff KM, Shi L and Kennelly PJ: The detection of enzyme activity following sodium dodecyl sulfate-polyacrylamide gel electrophoresis. Anal Biochem 260: 1-17, 1998.

16. Haycock JW: Polyvinylpyrrolidone as a blocking agent in immunochemical studies. Anal Biochem 208: 397-399, 1993.

17. Nishimori I, MinakuchiT, Onishi S, Vullo D, Cecchi A, Scozzafava A and Supuran CT: Carbonic anhydrase inhibitors. Cloning, characterization and inhibition studies of the cytosolic isozyme III with anions. J Enzyme Inhib Med Chem 24: 70-76, 2009. 